\title{
An open labeled study to evaluate safety and efficacy of Unicough syrup in children with acute bronchitis.
}

\author{
Dr. Sagar Ambekar ${ }^{1}$, Dr. Sarang Deshpande ${ }^{2}$, Patni Showkat ${ }^{2}$, Suhail Asghar ${ }^{2}$ \\ Dr. Shailesh Malekar ${ }^{2}$ \\ 1. Sanjivani Hospital Washim \\ 2 Unijules Life Sciences Ltd B - 35, 36 MIDC Area Kalmeshwar Nagpur - 441501 (Maharashtra) India
}

\begin{abstract}
Acute bronchitis is inflammation of the main air passage to the lungs generally follows a viral respiratory infection. Children generally suffer from acute bronchitis at any stage. Fumes, dusts, decreased immunity, indigestion, viral infections are some causes due to which air passage get inflamed and irritated. Acute bronchitis is a clinical syndrome produced by inflammation of the trachea, bronchi, and bronchioles. In children, acute bronchitis usually occurs in association with viral respiratory tract infection. Acute bronchitis is rarely a primary bacterial infection in otherwise healthy children.

In modern therapies using excessive expectorant, antihistaminics, and cough suppressants causes certain side effects and has no roll in rejuvenating the respiratory system. Unicough is a herbal remedy with the herbs which acts as a mucolytic and mild expectorant by its smoothening effect which helps in easy evacuation of cough and rejuvenate the respiratory passages. Current open labeled study on forty four children of acute bronchitis showed significant improvement after first and second week of the treatment in all associated symptoms which proves that Unicough syrup is a safe and effective remedy for acute bronchitis.
\end{abstract}

Keywords: Acute bronchitis, antihistaminic, rejuvenating, inflammation, expectorants.

\section{Introduction:}

Acute bronchitis is a common illness in the community. Although a source misery, the condition carries little risk of morbidity and almost none of mortality ${ }^{1}$. It is a common respiratory disease that usually affects all children in their childhood age. It is caused by chronic irritation of the respiratory tract by dust, and other atmospheric pollutants inhaled. In this condition, you will have chronic cough and expectoration of phlegm, especially during winter months ${ }^{2}$.

Herbal medicine is the leading alternative to conventional pharmaceutical treatment. When herbalist makes up a prescription for patients with asthma and bronchitis problems, they take into account all the factors which contribute to their health and try to improve all the systems which are affected by respiratory difficulties. This is called a holistic approach. It is the main difference between conventional and herbal treatment ${ }^{3}$.

Antibiotics are commonly prescribed, this type of therapy remains controversial, particularly since only half of all exacerbations are bacterial in origin, the rest being viral or chemical. Thus some believe that antibiotic therapy of acute exacerbation of bronchitis is an abuse of antibiotics, and can contribute to antimicrobial resistance ${ }^{4}$.

The natural approach to treating bronchitis avoids creating new disease problems. It involves two goals: stimulating the normal processes that expel mucus, and stimulating the immune system to deal with viral infection. Expectorant herbs increase the fluidity and volume of phlegm, and stimulate cough. They are not cough suppressants, since cough suppression traps phlegm in the bronchi and lungs ${ }^{5}$.

Unicough is a herbal remedy with Khaira Chhal (Acacia catechu), Vasakapatra (Adhatoda vasika), Vach (Acorus calamus), Elaichi (Elettaria cardamomum), Nagkeshar (Mesua ferrea), Talispatra (Abies webbina), Tulsi (Ocimum scantum), Dalchini (Cinnamomum zeylanicum), Sounth (Zingiber officinale), Pippali (Piper longum), Kababchini (Piper cubeba), Mulethi (Glycyrrhiza glabra), Somlata (Ephedra gerardiana), Dhatura panchang (Datura metel), Ajowan sat (Thymol), Pudina sat (Menthol). All these ingredients act as a smoothening, expectorant, bronchodilator and also improve the metabolism.

This was an open labeled, non comparative study in the patients of acute bronchitis for the period of 15 days.

\section{Materials And Methods:}

This was an open labeled, prospective, non comparative study conducted in the OPD of Sanjivani hospital Washim, in which forty four children between and including the ages of 3 to 12 years suffering from the symptoms of acute bronchitis i.e. dry or loose productive cough, chest pain, were selected for the study and the children suffering from any other chronic illness and requiring any other medication were excluded from the study. Prior informed consent of all the patients was taken before the start of the medication. 


\section{Study procedure:}

This was a 2 weeks trial, with the follow up visit after every 7 days. At the initial visit all the systemic examination, detailed ENT examinations, upper respiratory tract examination of all the patients were conducted. All the patients were investigated for hematological parameters i.e. hemoglobin $(\mathrm{Hb})$, total leukocytic count (TLC), differential leukocytic count (DLC), acute eosinophil count (AEC) at the start and the completion of the study.

All the patients who met the eligibility criteria were given Unicough syrup and advised to take 1 teaspoonful (5 $\mathrm{ml}$ ) twice/thrice a day after meal for the period of 2 weeks.

\section{Efficacy parameters:}

Primary efficacy parameters were reduction in symptomatic score at the end of the treatment period from the baseline values of sore throat, associated chest pain, fever, coughing, breathlessness, blocked nose and sinuses etc. secondary efficacy parameters were prevention of recurrence of the symptoms and occurrence of any adverse events.

\section{Follow-up and assessment:}

All the patients were called for follow-up after every 7 days for the period of two weeks and in each visit assessment of all the symptoms were done using four point scale of assessment of symptoms 0-Nil, 1-Mild, 2-Moderate, 3-Severe.

\section{Statistical analysis:}

Statistical analysis of the mean symptom score at the end of therapy compared with the baseline parameters was done using the paired' $t$ ' test.

Adverse events:

All the adverse events experienced by the patients and observed clinically were recorded and predefined as Unrelated, Probable and Possible.

All the patients were allowed for voluntary withdrawal from the study, if he experienced any discomfort or any other clinical event requiring medical care. Efforts were made to checkout the reason for the withdrawal.

\section{Results:}

Total fifty two patients were enrolled in the study out of which forty four patients have given complete follow up and others were drop out due to lack of follow up. Out of the forty four patients 28 children were male and 16 were female. The mean age of the children was $7+/-2.34$ (Table 1 ).

Table 1: Demographic data of the patients

\section{Demographic data of the patients}

\section{Parameters}

Age

Sex

Diet
Male

Female

Vegetarian

Mix
Observations

$$
7+/-2.34
$$

28

16

35

9

Extremely significant result was observed in all the symptoms of bronchitis. In case of sour throat mean symptom score before treatment was $1.272+/-0.094$ which was reduced to $0.11+/-0.048$ after two weeks of treatment period. Mean symptom score of fever was $1.363+/-0.152$ which was significantly reduced to $0.00+/-$ 0.00 means totally cured. Breathlessness was observed to be $1.590+/-0.087$ and after the end of treatment period it was $0.093+/-0.044$ means extremely significant. In case of associated chest pain mean symptom score before treatment was $1.454+/-0.157$ and after the end of two weeks treatment period it was significantly reduced to $0.272+/-0.140$. mean symptom score of coughing before treatment was $2.068+/-0.062$ and after the end of treatment period it was $0.272+/-0.067$ means reduced significantly. In case of blocked nose and sinuses also mean symptoms score was significantly reduced from $1.2+/-0.0816$ to $0.16+/-0.074$. This difference between before treatment and after first and second week of the treatment differs significantly because in all the cases $p$ is less than 0.0001 . 


\section{Symptoms}

Sour throat

Fever

Breathlessness

Associated chest pain

Coughing
Table 2:- Assessment of symptom score

before Tt. After 1W

$1.272+/-0.0940 .5+/-0.076^{* *}$

After 2W

$1.363+/-0.1520 .33+/-0.188 * * \quad 0+/-0.00 * *$

$1.590+/-0.0870 .43+/-0.075 * * 0.093+/-0.044 * *$

$\begin{array}{lll}1.454+/-0.157 & 0.363+/-0.152 * * & 0.272+/-0.140 * * \\ 2.068+/-0.082 & 0.840+/-0.085 * * & 0.272+/-0.067 * * \\ 1.2+/-0.0816 & 0.32+/-0.095 * * & 0.16+/-0.074 * *\end{array}$

Blocked nose \& sinuses $\quad 1.2+/-0.0816 \quad 0.32+/-0.095 * * \quad 0.16+/-0.074 * *$

IV. Discussion:

Acute bronchitis is an inflammation of the breathing passages called bronchi- usually because of a bacterial or viral infection. Acute bronchitis is a formal name for a cold that "goes to the chest" cough and chest congestion typically starts within a week of the cold. Acute bronchitis can also occur after exposure to a substance particularly irritating to your lungs ${ }^{6}$.

The vast majority of cases $(>90 \%)$ of acute bronchitis in otherwise healthy children and adults are caused by viral infections. Viruses that cause acute bronchitis include those that are responsible for lower respiratory tract symptom and those that result primarily in upper respiratory tract symptoms. Viral infections directly damage respiratory mucosa, increase mucus production, decrease mucociliary clearance, stimulate airway irritant receptors, and enhance airway reactivity. These inflammatory and physiologic changes give rise to symptoms of cough, sputum production and, in some cases, wheezing ${ }^{7}$.

Acute bronchitis is a frequent reason for visits to the healthcare provider, and one of the most common causes of inappropriate prescribing of antibiotics ${ }^{8}$. Ninety percent of patients with acute bronchitis have a viral illness and require no more than reassurance and symptomatic treatment. Patients with acute bronchitis do not benefit from antibiotics because bacteria are rarely responsible ${ }^{9}$. Other reviews have failed to find any benefits from antibiotic use ${ }^{10-12}$. Assuming that, at best, there is a modest benefit of antibiotics and that there is a concern about developing antibiotic resistance, the routine use of antibiotics is not recommended for individuals with acute bronchitis who are otherwise healthy ${ }^{13}$.

Other allopathic remedies for bronchitis include cough suppressant, antihistaminic, expectorants etc. which has following side effects.

Young children are more susceptible to centrally acting cough suppressant like codeine intoxication due to their immune hepatic glucuronidation system ${ }^{14}$. Adverse reaction of codeine in nonintoxicated children includes nausea and vomiting, constipation, palpitation and dizziness ${ }^{14}$. Side effects of the antihistaminic include central nervous system stimulation, extrapyramidal symptoms, antimuscarinic effects, and gastrointestinal disturbance $^{15}$. Expectorants may produce gastric irritation and vomiting.

Unicough syrup is a balanced remedy with the herbs having smoothening, expectorant, and digestive activity as poor digestion is needed to be rule out for proper evaluation of acute bronchitis. Unicough is beneficial in both productive and dry cough. Its peripheral antitussive action reduces the bronchial mucosal irritation. Its anti-inflammatory activity reduces inflammation in the upper respiratory tract, especially in the pharynx and larynx. In addition, the anti-allergic, antimicrobial and immune-resistance building properties provide relief from cough of varied etiology. The key herbs in it has following action

Khair chhal (Acacia catechu) is useful in throat and tonsillar affections ${ }^{16}$. Vasa patra (Adhatoda zeylanicum) is a bronchodilatory and expectorant. Dilates small bronchioles of lungs and allow the bad cough to release out ${ }^{17}$. Mulethi (Glycyrrhiza glabra) is anti-inflammatory and it reduces inflammation of the respiratory $\operatorname{tract}^{18}$. Vach (Acorus calamus) is useful in relieving bronchial spasm. Elaichi (Elettaria cardamomum) is bronchodilator and useful in upper respiratory tract infections, it is digestive and appetizer. Nagkeshar (Mesua feria) is anti-inflammatory and antipyretic. Talispatra (Flacourtia jangomas) is useful in bilius affections. Tulsi (Ocimum sanctum) is expectorant which helps in expelling cough and soothens the respiratory system. Dalchini (Cinnamomum tamala) is bronchodilator and helps in releasing the cough from respiratory passage. Sounth (Zingiber officinale) is useful in cold and other bronchial affections; it reduces inflammation and congestion of the respiratory tract. Pippali (Piper longum) prevents recurrent attacks of bronchial asthma. Kababchini (Piper cubeba) is expectorant. Ajowan sat (Menthol) is mucolytic agent, it protect the mucous membrane without increasing bronchial secretion, and produces a soothing effect. Thus overall balanced action of Unicough syrup is soothing, mild expectorant and rejuvanative to the respiratory system. 
Current study also shows significant improvement in all the aspects of the acute bronchitis even after one week of the treatment, and without causing any adverse events etc. even no evidence of feeling drowsiness etc. was observed during and after the therapy. Hence it can be conclude that Unicough syrup is a potent remedy to relieve acute bronchitis.

\section{References:}

[1] Edward Ringel, The Little Black Book of Pulmonary Medicine; 2009, 239

[2] Savitri Ramaiah, All You Wanted To Know About Chronic Bronchitis; 2000, 6.

[3] Jill Wright, Asthma \& bronchitis; 2002, 37.

[4] Luigi Allegra, F. Blasi Mechanisms and management of COPD exacerbations, 2000; 73

[5] Phyllis A. Balch; Prescription for Herbal Healing: An Easy-to-Use A-Z Reference to Hundreds of common disorders and their remedies, 2002;123

[6] Patricia Carroll; What Nurses Know and Doctors Don't Have Time to Tell You 2004; 322

[7] Michael A. Kaliner; Current review of asthma 2003; 83

[8] Gonzales R, Steiner J F, Sande MA. Antibiotics prescribing for adults with colds, upper respiratory tract infections, and bronchitis by ambulatory care physicians. JAMA 1997;(11):901-4

[9] Phyllis Carolyn Leppert, Jeffrey F. Peipert Primary Care for Women; 2004; 378

[10] Graf PM. Rhinitis medicamentosa. Clin Allergy Immunol 2007;19:295-304.

[11] Passali D, Salerni L, Passali GC, Passali FM, Bellussi L. Nasal decongestant in the treatment of chronic nasal obstruction: efficacy and safety of use. Expert Opin Drug Saf 2006;5(6):783-90.

[12] Ferguson BJ, Paramaesvaran S, Rubinstein E. A study of the effect of nasal steroid sprays in perennial allergic rhinitis patientswith rhinitis medicamentosa. Otolaryngol Head Neck Surg 2001;125(3):253-60.

[13] Tekoa L. King, Mary C. Brucker; Pharmacology for Women's Health; $2011 ; 571$.

[14] Olson Al, Klein SW, Charney E, Mac Whinney JB, Mclnemy TK, Miller RL. Prevention and therapy of serious otitis media by oral decongestant: a double blind study in pediatric practice. Pediatrics 1978; 61(5):679-84.

[15] Opioid analgesics. In: Reynolds JEF, ed, Martindale. The Extra Pharmacopeia. 30 ${ }^{\text {th }}$ ed. London, The pharmaceutical press, 1993;1065-98.

[16] Narain Singh Chauhan Medicinal and Aromatic Plants of Himachal Pradesh; 1999; 55

[17] A. K. Mehta, Herbal Remedies for Asthma; 2002; 80

[18] C.M. Ghai; Health Rejuvenation And Longevity Through Ayurveda; 2004; 183 